\title{
Development of a Face Detection Algorithm Based on Skin Segmentation and Facial Feature Extraction
}

\author{
Jide J. Popoola**, Akintunde Akinola* \\ *Department of Electrical and Electronics Engineering, Federal University Technology, P.M.B. 704, Akure, Ondo State, \\ Nigeria \\ (jidejulius2001@gmail.com, akinolatunde11@gmail.com)
}

${ }^{*}$ Corresponding Author; Jide J. Popoola, Department of Electrical and Electronics Engineering, Federal University of Technology, P.M.B. 704, Akure, Ondo State, Nigeria. Tel: +234803 413 1860,

jidejulius2001@gmail.com

Received: 27.03.2017 Accepted: 23.08.2017

\begin{abstract}
This paper presents a face detection algorithm capable of detecting face(s) without prior training as a face classifier. The technique employed in developing the algorithm is based on skin segmentation and facial feature extraction of the two eyes and mouth. Skin segmentation was done in the red, green, blue color space. White balance correction was employed to correct the change in image temperature that occurs due to change in lighting conditions at the point of acquiring image. Morphological operations and bounding box were employed to search and extract face region from the segmented skin region. Facial feature, eyes and mouth, were extracted for final verification of the sensed face using the Laplacian of Gaussian filter and the isosceles triangle matching rules. The extracted features were used to develop the face detection algorithm. The developed algorithm was evaluated using random images taken under different lighting conditions. Furthermore, the efficiency of the developed face detection algorithm was evaluated using a standard face detection image database. The result obtained shows that the developed face detection algorithm performed satisfactorily well with $81.37 \%$ detection accuracy. Furthermore, the results obtained from the performance evaluation of the developed face detection for this study has shown it clearly that accuracy detection of dissimilar faces in images with complex background is possible and attainable.
\end{abstract}

Keywords Face detection, face detection methods, skin segmentation, morphological operations, isosceles triangle matching.

\section{Introduction}

In image processing, in image processing, one of the current research areas is automatic face detection. Interest in automatic face detection is increasing on daily basis because of countless applications that require the application of automatic face detection as a cornerstone for series of applications revolving around automatic facial image analysis [1]. For instance, as reported in [2], it is being used in applications such as authentication, attendance system and electronic passport. In addition, as reported in [2], it has also been used in a new naked-eye auto-stereoscopic display which requires accurate human face and eye locations. According to [1], face detection is also the initial step towards most of the modern vision-based human-computer and human-robot interaction systems. Basically, face detection in real-world is one of the visual tasks that human beings can do effortlessly. However, in computer vision, human faces detection is a challenging task for computers. This is because human faces are not easy to model as face modeling according to [3] requires accounting for all possible appearance variations caused by changes in scale, location, orientation, pose, facial expression, lighting conditions and partial occlusions.

Tactically, the main task of face detection system or algorithm is to determine whether or not a face exists in a given image. Hence, according to [4], face detection is defined as a process of determining whether a face is present or not in a scanned or digitalized photo, an image or a video, and reporting the location of each face(s) if there are any. It is the first step of automatic system that analyzes the information contained in faces. Face detection plays an important role in success of any face processing system. Often, face recognition systems work by applying a face 
detector to locate the face, and then apply a separate recognition algorithm to identify the face [5].

Basically, process of automatic human face recognition is one of the most difficult and important areas in computer vision and pattern recognition. It is as a security mechanism being used in replacing metal key, plastic card, and password or PIN number. However, most face recognition systems or algorithms require the input face to be free of background, that is, to be separated from the entire image. Therefore, a successful face detection process is essential in enabling successful face recognition task. If we do not have a successful face detection method, we cannot develop a successful face recognition system [6]. Therefore, face detection process should not be treated merely as a preprocessing of a face recognition system or other computer vision process; rather, it should be treated with the same importance as the other computer vision problems.

Conventionally, according to [7-10] face detection techniques or methods are classified into four classes: knowledge-based methods, feature invariant methods, template matching methods, and appearance-based methods. In knowledge-based methods, human knowledge of typical face geometry and arrangement of facial features are usually employed. In this method, facial features in an input image are first extracted, which aids face candidate's identification based on coded rules. On the other hand, in feature invariant methods, structural features that exist in the face, such as facial local features, texture, shape, and skin color, are usually targeted and use to locate faces [5]. In template matching methods, a standard face pattern is usually parameterized by a function. Hence, this method involves computation of the correlation values between the standard pattern and an input image. While face detection algorithms based on template matching methods depend on a predefined template to function, appearance-based face detection algorithms learn from examples in images. In this face detection method; face detection is usually view as a pattern classification problem with two subsets: face and non-face.

Based on these four methods, a lots of face detection algorithms have been developed. Similarly, in detecting face, various skin color based algorithms have been developed. The adoption of skin color based algorithm is based on the fact that color is an important feature of human faces [11]. According to [11], using skin color as a feature for locating a face has diverse advantages. One of these advantages, according to [12], is the fact that color processing is faster than other facial features processing. In this paper, a skin color segmentation based on feature invariant face detection approach to extract the skin areas in single image was employed. The method works by checking the pixels in order to categories pixels into skin and non-skin. The rule governing the pixel's classification employed is stated in Section 3. For sequential and logical presentation of the study presented in this paper, the rest of this paper is structured as follows. Section 2 presents review on related work on face detection algorithms. In Section 3, detailed information on the procedures involved in developing the face detection algorithm for this study is presented. The performance evaluations result of developed face detection algorithm for this study is presented and discussed in Section 4. The paper is finally concluded in Section 5.

\section{Related Work}

There are many researches on face detection using different methods that have been carried out over the years. Those that were centered on skin segmentation in detecting faces in images are reviewed in this section. In [13], skin like pixel was detected using evolutionary agents. In the study face like regions were segmented from the skin-like pixel by activating the evolutionary behavior of the agents. Then wavelet decomposition was applied to each region to detect the possible facial features and a three-layer back propagation neural network (BPNN) was used to detect eyes among the features. Similarly, in [11], facial feature was used to detect faces. Skin like region was first segmented using a combination of color space. Eyes and mouth were then extracted using principal component analysis on the detected skin like region. Likewise, in [14], segmented skin like region with the Hue saturation value color space were employed. Feature vector of the segmented skin like region was calculated with two dimensional discrete cosine transform. BPNN was used to classify the feature vectors that belong to skin like regions. Similarly, authors of Reference [15] also performed skin color segmentation for skin like regions using the Luminance-Blue Chroma Difference Red Chroma Difference (YCbCr) color space and possible faces were found using Linear Support Vector Machine (SVM). For final verification of face, eyes and mouth are verified using the information gotten from $\mathrm{Cb}$ and $\mathrm{Cr}$. They found eye and mouth via the difference in value of $\mathrm{Cb}$ and $\mathrm{Cr}$. For eye region; $\mathrm{Cb}>\mathrm{Cr}$ and for mouth region; $\mathrm{Cb}<\mathrm{Cr}$, where $\mathrm{Cb}$ is the value of Blue Difference Chroma and $\mathrm{Cr}$ is the value of Red Difference Chroma. Likewise, authors of Reference [16] also performed segmented skin like regions using the Luminance-Blue Luminance Difference-Red Luminance Difference (YUV) color space to decrease computational time when using the window scanning technique. The window just scans the segmented region instead of scanning the whole image. The segmented skin like regions is sent to BPNN to find face candidates. The authors in [16] carried out the final verification of face using Bayesian Decision

Aping et al (2010), [17], modeled skin color in the elliptical region using $\mathrm{Cb}$ and $\mathrm{Cr}$ channel in $\mathrm{YCbCr}$ color space. Skin like regions are segmented if the color values are clustered inside the elliptic region. Face is verified by using template matching. Li et al (2010), [18], performed skin color segmentation with the threshold values of $\mathrm{Cb}, \mathrm{Cr}$, normalized $\mathrm{r}$ and normalized $\mathrm{g}$. Faces are then selected from the skin like region based on the following criteria; bounding box ratio, ratio of area inside and area bounding box, and minimum area of the region. The final verification is done by combining the results from skin like region with AdaBoost.

In most of these algorithms, two profound costly assumptions are usually made by the developers [19]. The first assumption according to [19] is to assume the availability of frontal faces of similar sizes, which in reality 
is not generally true due to varied nature of face appearance and environmental conditions. The second assumption usually made by those developers has observed by [19] is that the exclusion of background in their images is necessary for reliable face detection. However, in most situations, a face could occur in a complex background hence making this assumption invalid. Similarly, most of the developed face detection algorithms described requires the algorithms to be trained first as a face classifier. This training takes several hours or even days to obtain accurate result. Although some face detector or algorithms only needs to be trained once to obtain the trained data, if the trained data is lost or damaged this time consuming training process have to be carried out all over again. These observed disadvantages necessitate the development of the face detection algorithm developed in this study using feature invariant approach. The method employed in this study detects faces without prior training of the developed face detection algorithm as a face classifier. This is done to save time that would have been consumed in training the algorithm as well as reducing the algorithm computational complexity. The step-by-step approach in developing the face detection algorithm for this study is presented in next section.

\section{Developed Face Detection Procedural Stages}

In developing the face detection algorithm for this study, whose goal is to detect the presence of faces in an image, the activities involved were divided into: white balance correction performance, segmentation of skin color in the red, green, blue (RGB) color space, logical 'and' operations, morphological operations and application of bounding box, face detection verification by extracting the facial feature of eyes and mouth, and application of isosceles triangle matching rules. The flow of the all activities in the framework is shown in Fig. 1. Detailed information on each stage involved in developing the face detection algorithm for this study as well as the results obtained in some stages, where appropriate, are presented and discussed for more clarification in the following subsections.

\subsection{White Balance Correction}

This is the first stage involved in developing the face detection algorithm for this study. The white balance correction was performed to correct variation in white balance of the image as a result of differences in illumination conditions of the environment where an image is acquired, which might cause some non-skin region look like skin region. Therefore, it is important to correct the white balance of input image before segmentation is performed. Also, it is important to carry out white balance correction process before segmentation because it enhances the production of good output of skin color and improves the overall detection rate.

The white balance correction was performed as follows: The average value of red channel $\left(\mathrm{R}_{\mathrm{av}}\right)$, green channel $\left(\mathrm{G}_{\mathrm{av}}\right)$, and blue channel $\left(\mathrm{B}_{\mathrm{av}}\right)$ of image is first obtained. The average gray value of the image is then calculated from the obtained channel values information using the mathematical expression presented in [20];

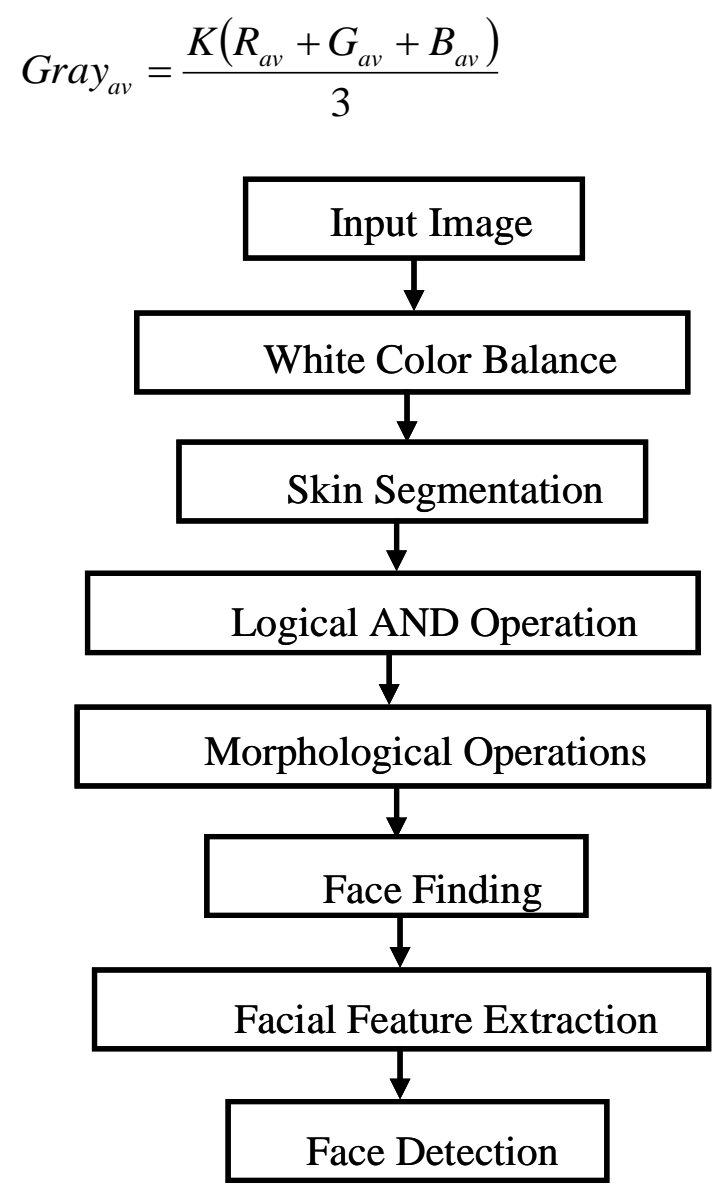

Fig. 1. The framework of the developed face detection.

The obtained average gray value is then used to calculate an adjustment parameter for each color channel using the following relationships;

$$
\begin{aligned}
& K R=\frac{G r a y_{a v}}{R_{a v}} \\
& K G=\frac{G r a y_{a v}}{G_{a v}} \\
& K B=\frac{G r a y_{a v}}{B_{a v}}
\end{aligned}
$$

where $K R, K G$ and $K B$ are the adjustment parameters for red, green and blue channels respectively. The adjustment parameter that is obtained is used to generate a new image, New (I), from original image, Org (I), using the relationships presented in [20];

$$
\begin{aligned}
& \operatorname{New}(R)=K R \times \operatorname{Org}(R) \\
& \operatorname{New}(G)=K R \times \operatorname{Org}(G)
\end{aligned}
$$




$$
\operatorname{New}(B)=K R \times \operatorname{Org}(B)
$$

Typical original input image and the resulting image after white balance correction in this study is shown in Fig. 2a and Fig. 2 b respectively.

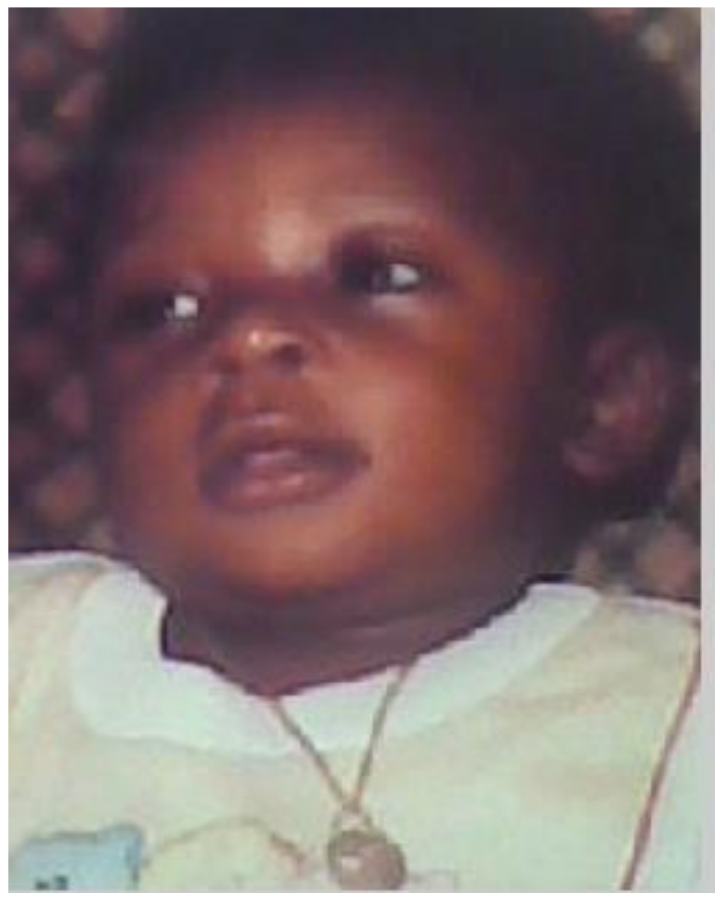

(a)

Fig. 2a. Original input image.

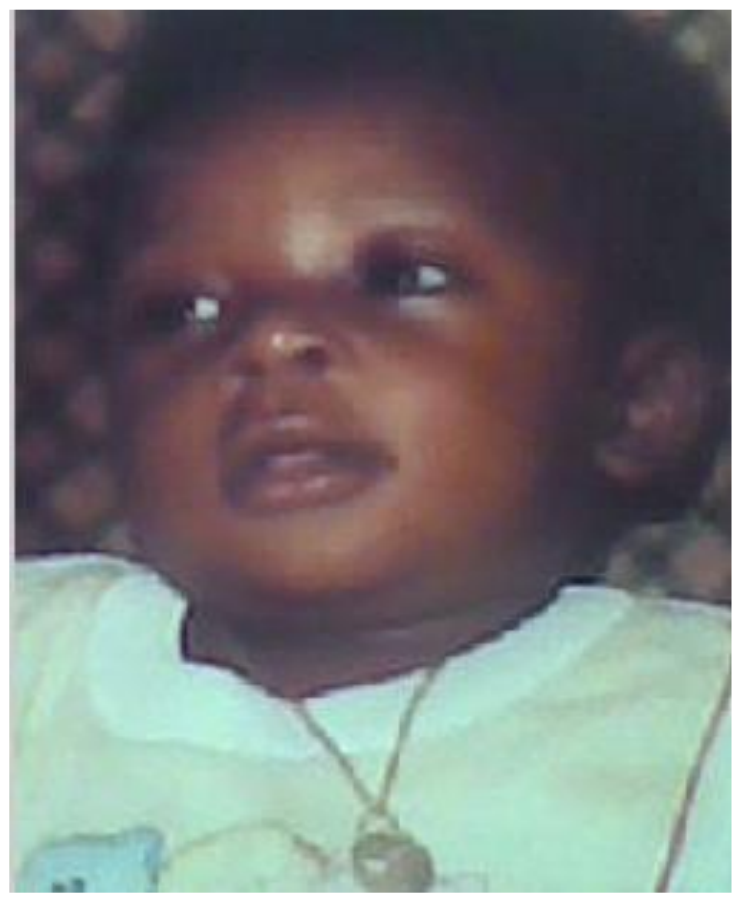

(b)

Fig. 2b. White balance correction image.

\subsection{Skin Segmentation}

Skin color segmentation is the second step involved in developing the face detection algorithm for this study. This step is important because only the segmented skin region is searched while searching for face. It therefore helps in reducing computational time in face detection algorithm development. In developing the face detection algorithm for this study, the RGB color space technique was used in describing skin color. The skin segmentation was performed based on the rules for skin segmentation in RGB space reported in [5,21], which is defined as;

$$
\text { pixel }=\left\{\begin{array}{cl} 
& R>95 \text { and } G>40 \\
& \text { and } B>20 \text { and } \\
\text { skin, } & \max \{R, G, B\}- \\
& \max \{R, G, B\}>15 \\
& |R-G|>15 \text { and } R>G \\
& \text { and } R .>B \\
\text { non-skin } & \text { otherwise }
\end{array}\right.
$$

The parameters $\mathrm{R}, \mathrm{G}$ and $\mathrm{B}$ are the red, green and blue channel value of the color pixel of image. If the pixel satisfies the conditions stated in (Eq. 8), it is regarded as skin color and binary image is formed, thus the algorithm moves to the next stage.

\subsection{Logical AND Operation}

The logical 'AND' operation is the third stage involved in developing the face detection algorithm for this study. The stage is essentials for two reasons: (i) to eliminate generated unwanted skin color like region that occasionally occurs during white balance correction from both the segmented original image and segmented; and (ii) to extract the final skin image. Fig. 3 shows the final skin image obtained from the logical AND operation performed on the original and white balance corrected image in this study.

\subsection{Morphological Operations}

Morphological operations are the fourth stage involved in developing the face detection algorithm for this study. Morphology, according to [22], is a broad set of image processing operation that process images based on shapes. Its objective is to transform the images into simpler ones by removing irrelevant information while essential shapes of the images are reserved. Thus, it helps in enhancing the image so as to make face finding in the image easy. The morphological operation applied at this stage in this study is the morphological closing operation. The closing operation merges gaps and fills holes. The morphological closing operation is achieved by applying dilation and erosion operation using the same structural element. According to [23], while dilation adds pixels to the boundaries of objects in an image, erosion removes pixels on object boundaries. After the successful completion of the stage, the face finding stage was carried out. 


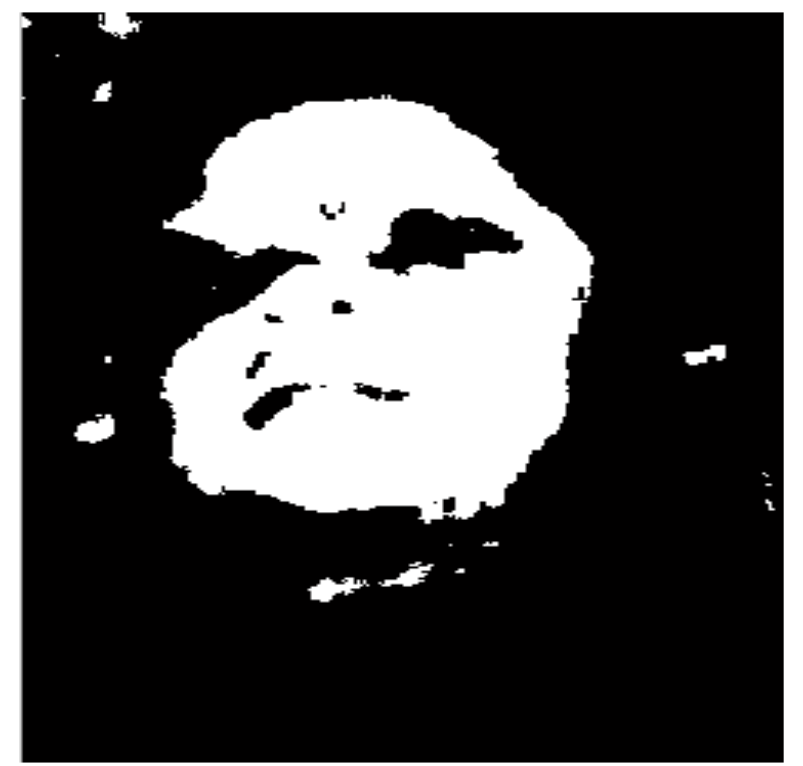

Fig. 3. Segmented skin region image.

\subsection{Face Finding}

Face finding is the fifth stage in the development of face detection for this study. The stage is the next stage after morphological operations, which is to analyze all the skin regions to determine if it is a face region or not. Every skin region is labeled as shown in Fig. 4a. On each label two conditions are considered to select a region to be a face region. The first condition is the ratio of bounding box covering each label. This ratio, which is defined as the width over the height of the bounding box, must lie between 0.6 and 1.2. This is in accordance with [24] that states that the ratio of width to height of the region of interest is usually 1 . The other condition is to cover some gaps inside the region in order to distinguish the face from the other body part such as hand as segmentation on hand will have no gaps which makes it different from face. Based on these two conditions, the face region is selected and is extracted from the input image with the bounding box as shown in Fig. 4b. The extracted face is then sent to the facial feature extraction stage for final verification of face.

\subsection{Face Feature Extraction}

This is the sixth and last stage of the algorithm development. Facial features are significant features of the face. Examples of facial features are eyes, eyebrows, nose, lips, mouth, cheek, etc. In this study, the facial features that were extracted are the two eyes and the mouth. Laplacian of Gaussian (LoG) filter was applied to enhance the image. The essence of the filtering process is to define the features to be extracted as well as detecting edges so as to properly separate the region of interest from the background.

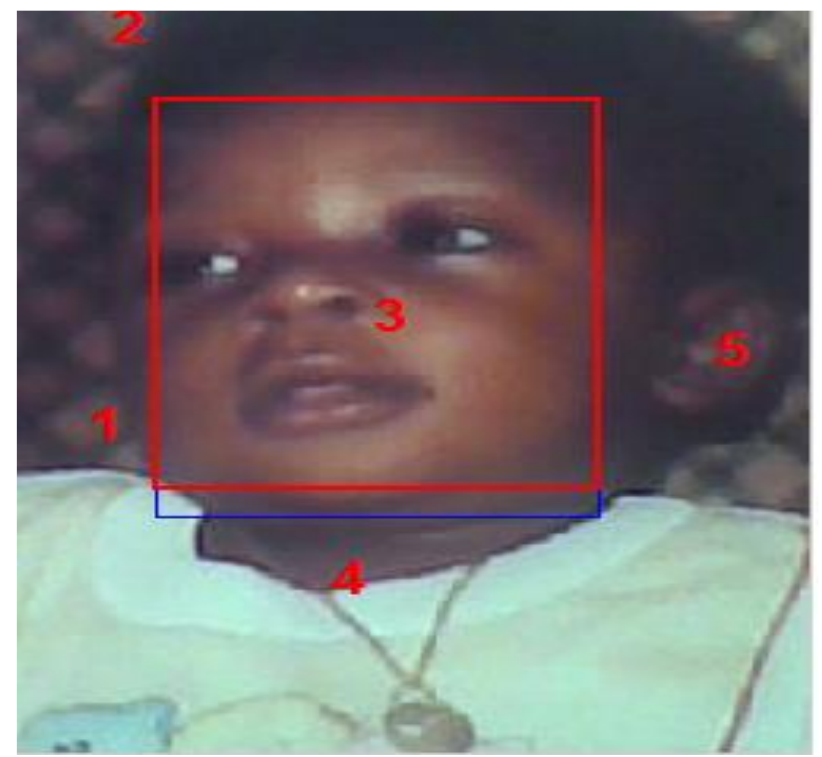

Fig. 4a. Bounding box on the face region.

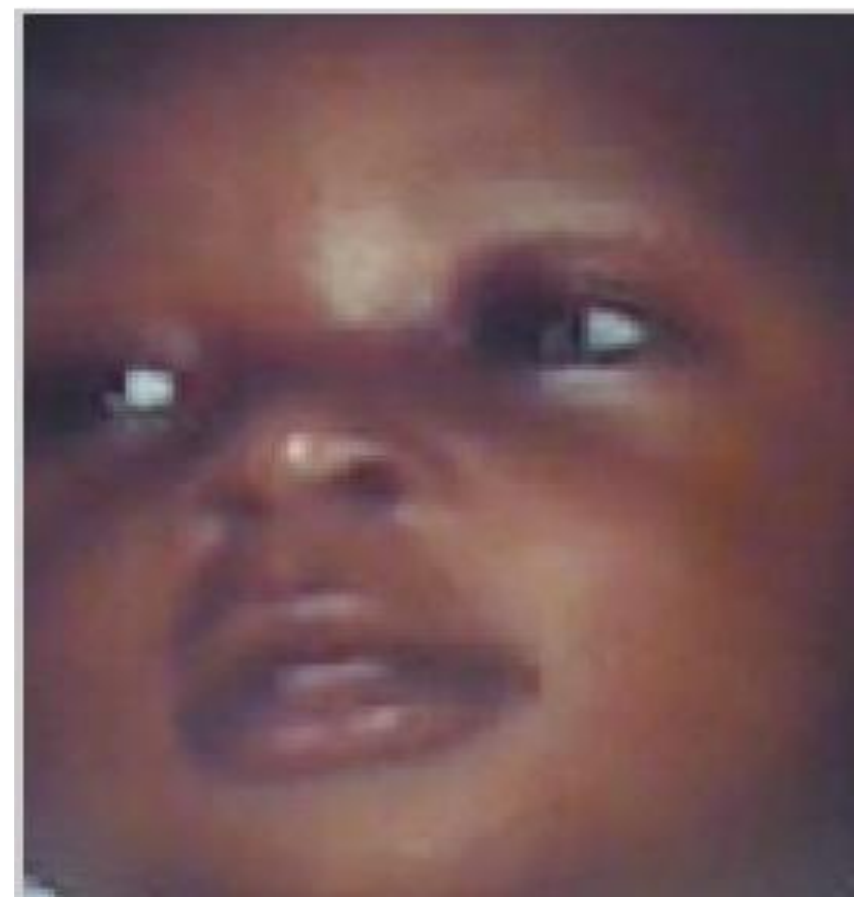

(b)

Fig. 4b. Face region from bounding box.

The Laplacian of Gaussian combines Gaussian filter with Laplacian for edge detection. The Laplacian detects the edge point of the image by finding the zero crossing of the second derivative of the image intensity. However, because the second derivatives are very sensitive to noise, there is the need for Gaussian filter to swipe away noise before filtering. Since LoG filter is linear, the two constituent filters (Gaussian and Laplacian) can be applied separately. The operation can be performed by convolving the image with a Gaussian smoothening filter and then computing the Laplacian of the result using mathematical expression in (Eq. 9);

$$
L(x, y) \otimes G(x . y) \otimes f(x, y)
$$


where L, G and $f$ are the Laplacian, Gaussian and Image respectively. The result of the filtering operation of a found face region is shown in Fig. 5.

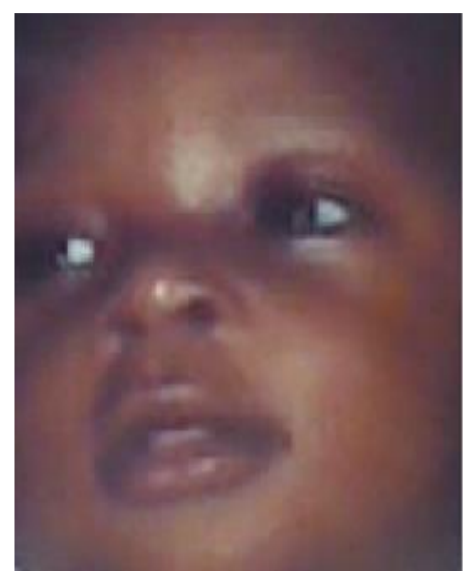

(a)

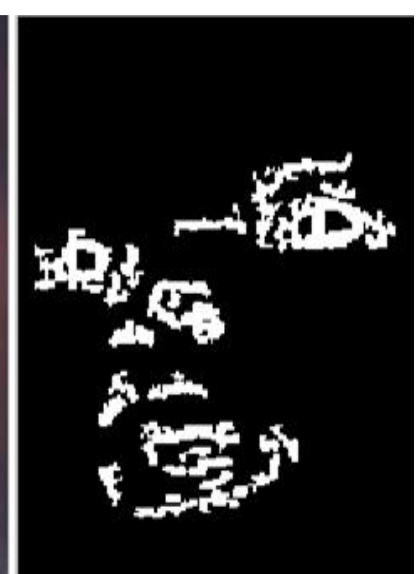

(b)
Fig. 5. (a) Found face region (b) LoG filtered image.

The application of the LoG filter helps to define facial features properly. With this, the facial feature of eyes and mouth can be extracted. The image resulting from the LoG filter is divided into three regions of right (R), left (L) and down (D) regions as shown in Fig. 6. The right eye is detected in the right region and the left eye in the left region by using the following criteria according to [25];

(i) The $\mathrm{X}$ position of right eye should be in region of $0.125 \times$ width to $0.405 \times$ width;

(ii) The $\mathrm{X}$ position of left eye should be in region of $0.585 \times$ width to $0.865 \times$ width;

(iii) The Y position of left eye and right eye should be less than $0.5 \times$ height;

(iv) Area should be greater than 100 pixel square; and

(v) Bounding box ratio of label should be in the region of 1.2 to 4

where width in the criteria denotes the width of the face image and height denotes height of face image [25].

If a label in the right region satisfies criteria (i), (iii), (iv) and (v), it is selected to be a right eye. Similarly, if a label in the left region satisfies criteria (ii), (iii), (iv) and (v) then it is selected to be the left eye. If right eye and left eye is selected successfully, mouth can be selected using the isosceles triangle matching rule. According to [26], the two eyes and mouth generate an isosceles triangle and also the distance between the two eyes and the midpoint of the eyes to mouth are equal. Any component of the down region can pass for mouth candidate. Therefore, to find mouth the following steps were taken.

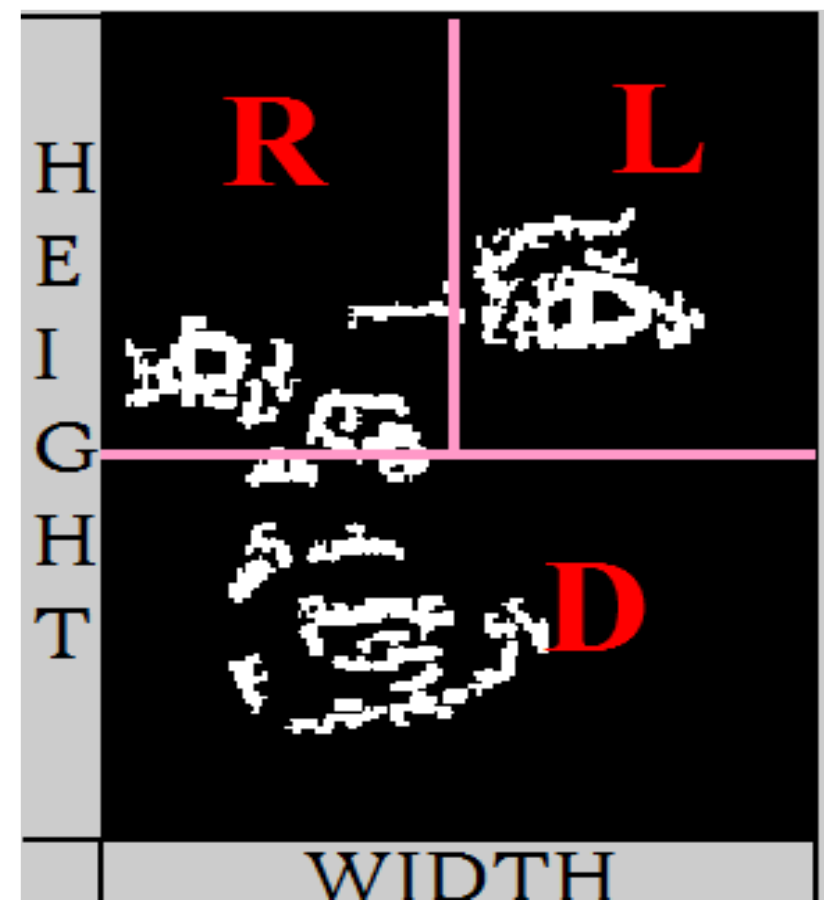

Fig. 6. Division of filtered image into three regions.

If a label in the right region satisfies criteria (i), (iii), (iv) and (v), it is selected to be a right eye. Similarly, if a label in the left region satisfies criteria (ii), (iii), (iv) and (v) then it is selected to be the left eye. If right eye and left eye is selected successfully, mouth can be selected using the isosceles triangle matching rule. According to [26], the two eyes and mouth generate an isosceles triangle and also the distance between the two eyes and the midpoint of the eyes to mouth are equal. Any component of the down region can pass for mouth candidate. Therefore, to find mouth the following steps were taken.

Euclidean distance of right eye to a possible mouth $\left(\mathrm{R}_{\mathrm{x}}\right)$ and Euclidean distance of left eye to a possible mouth $\left(\mathrm{L}_{\mathrm{x}}\right)$ is calculated. Euclidean distance between the midpoint of eyes and the possible mouth $\left(\mathrm{M}_{\mathrm{x}}\right)$ is calculated and lastly Euclidean distance between the two eyes $\left(\mathrm{E}_{\mathrm{x}}\right)$ is also calculated. From the calculated distance, the mouth was found by determining the absolute difference between $\mathrm{R}_{\mathrm{x}}$ and $L_{x}$. and the absolute difference between $E_{x}$ and $M_{x}$. The sum of the two differences is used in determining the total error $\mathrm{T}_{\mathrm{e}}$ using the mathematical expressions in (10), (11) and (12) respectively.

$d_{1}=\left|R_{x}-L_{x}\right|$

$d_{1}=\left|E_{x}-M_{x}\right|$

$T_{e}=d_{1}+d_{2}$

where $d_{1}$ and $d_{2}$ are the absolute differences between $\mid R_{x}$ and $L_{x} \mid$ and $\mid E_{x}$ and $M_{x} \mid$ respectively. If the values of $d_{1}$ and $d_{2}$ are less than 0.25 of the Euclidean distance between the two eyes $K\left(E_{x}\right)$, then the component is possibly a mouth. The component with the minimum total 
error $K\left(T_{e}\right)$ is selected as mouth. If mouth is found it means the facial features, eyes and mouth which are the required features to be extracted has been found. On the basis of the found facial features, verified face image is extracted. Fig. 7a and Fig. 7b show the isosceles matching of the two eyes and mouth on the image and the extracted final image obtained respectively.

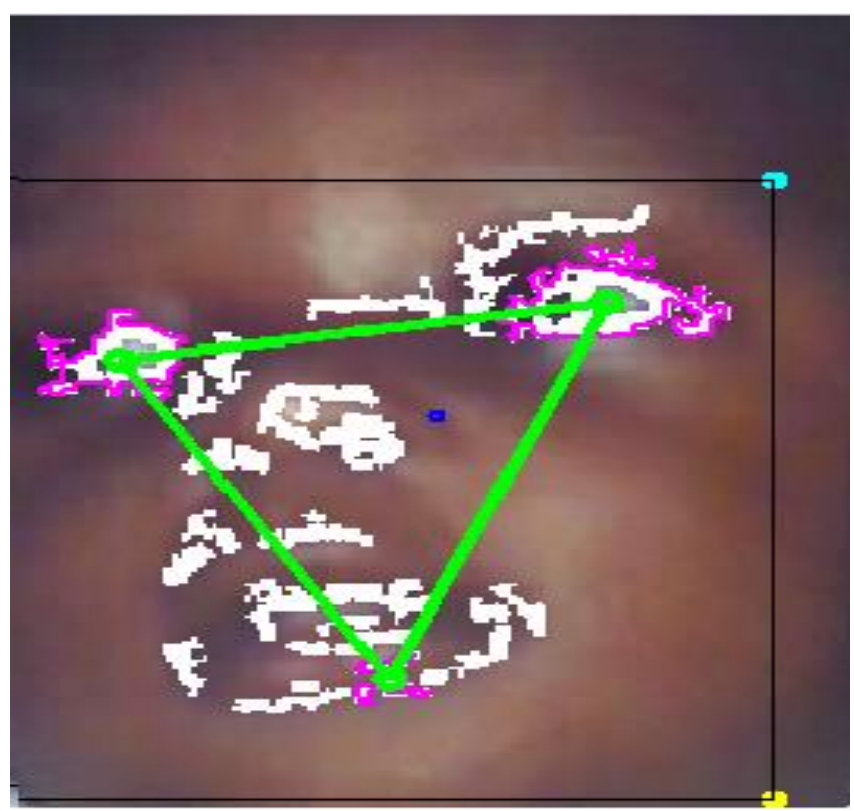

(a)

Fig. 7a. Extraction of facial feature.

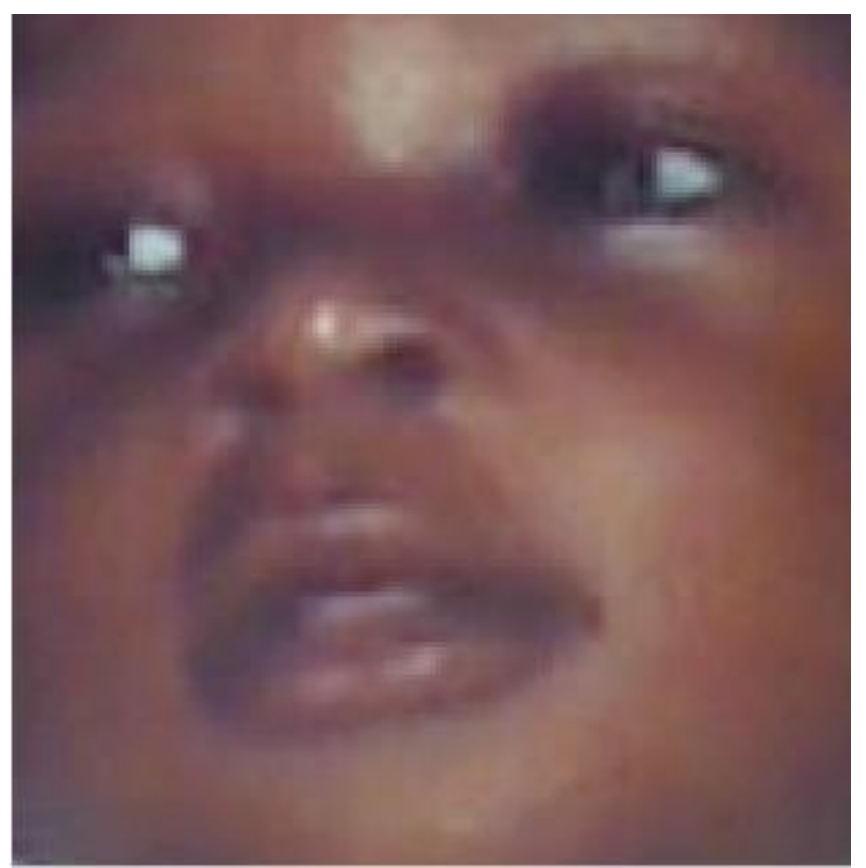

(b)

Fig. 7b. Detected face image.

\section{Developed Face Detection Algorithm Performance Evaluation}

After the successful development of the face detection algorithm, its performance evaluation was carried out. In order to present and discuss the performance evaluation of the developed face detection algorithm satisfactorily, this section is divided into two subsections. In the first subsection, the actual detection accuracy of the algorithm was evaluated using three different performance indices. In the second subsection, the comparative performance evaluation of the developed algorithm was carried out by comparing its result with other related studies in the surveyed literature.

\subsection{Developed Algorithm Detection Accuracy Evaluation}

The developed algorithm's detection accuracy performance evaluation was conducted using the Bao face database. The database contains a total of 370 color images. 149 of the images contain just one person while the remaining 221 contain more than one person. The primary purpose of the database is for measuring face detection algorithm. Images in this database were taken under uncontrolled conditions; i.e. uncontrolled illumination, varying pose, different facial expression for both outdoor and indoor images. From this database 102 images were selected randomly to evaluate the performance of the algorithm. Images were also selected randomly to test the performance of the algorithm using the following parameters: true positive (TP), which are the total numbers of faces correctly detected as faces, true negative (TN), which are the total numbers of non-faces correctly detected as non-faces and false positive (FP), which are the total numbers of non-faces wrongly detected as face. The performance indices employed in evaluating the developed algorithm are the correct detection rate (CDR), miss rate (MR) and false detection rate (FDR). In estimating the performance indices, the mathematical expression used are as follows;

$$
\begin{aligned}
& C D R=\frac{T P}{T F} \times 100 \% \\
& M R=\frac{T N}{T F} \times 100 \% \\
& F P R=\frac{F P}{T F} \times 100 \%
\end{aligned}
$$

where $T F$ is the total number of faces. The result of the measured detection accuracy for the developed algorithm is presented in Table 1 . 
Jide Julius Popoola et al., Vol.3, No.3, 2017

Table 1. Detection rate accuracy result

\begin{tabular}{|c|c|c|c|c|c|c|c|}
\hline \multirow[t]{2}{*}{ Image used } & \multirow{2}{*}{$\begin{array}{l}\text { Number } \\
\text { of image }\end{array}$} & \multicolumn{3}{|c|}{ Without white balance correction } & \multicolumn{3}{|c|}{ With white balance correction } \\
\hline & & $\operatorname{CDR}(\%)$ & $\operatorname{MR}(\%)$ & FPR $(\%)$ & $\operatorname{CDR}(\%)$ & $\operatorname{MR}(\%)$ & FPR $(\%)$ \\
\hline Bao-Database & 102 & 71.57 & 28.43 & 2.94 & 81.37 & 18.62 & 2.94 \\
\hline $\begin{array}{l}\text { Selected } \\
\text { Random Image }\end{array}$ & 15 & 73.33 & 26.67 & 0.00 & 80.00 & 20.00 & 0.00 \\
\hline
\end{tabular}

Table 1 shows the correct detection rate, miss rate and false positive rate for the developed algorithm with and without white balance correction. The Table shows that with white balance correction, the detection rate of the algorithm improves from $71.57 \%$ when there was no white balance correction to $81.37 \%$ when there was white balance correction for the Bao database. Similarly, for the images taken at random, there was an increase in detection rate from $73.33 \%$ to $80 \%$ when there was no white balance correction and when there was white balance correction respectively. The overall result shows that the developed algorithm performs satisfactory well with an average correct detection rate that is over $70.0 \%$ with and without white balance correction.

In addition, the detection accuracy of the developed algorithm for this study was further evaluated using a group photograph from Bao-database that consists of dissimilar frontal face sizes as well as complex background as shown in Fig. 8a and Fig. 8b. The obtained results of the face finding and face feature extraction analyses and typical isosceles checking as well as some typical faces detected when Fig. 8a and Fig. $8 \mathrm{~b}$ were used to evaluate the performance of the developed algorithm are shown in Fig. 9a-d. Three out of the images in Fig. 8a and Fig. 8b were selected for final analysis in Fig. 9c and Fig. 9d because of space.

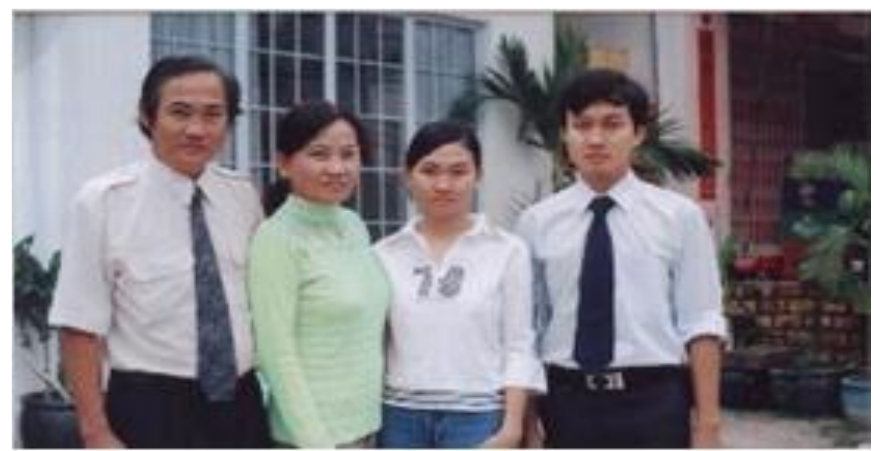

(a)

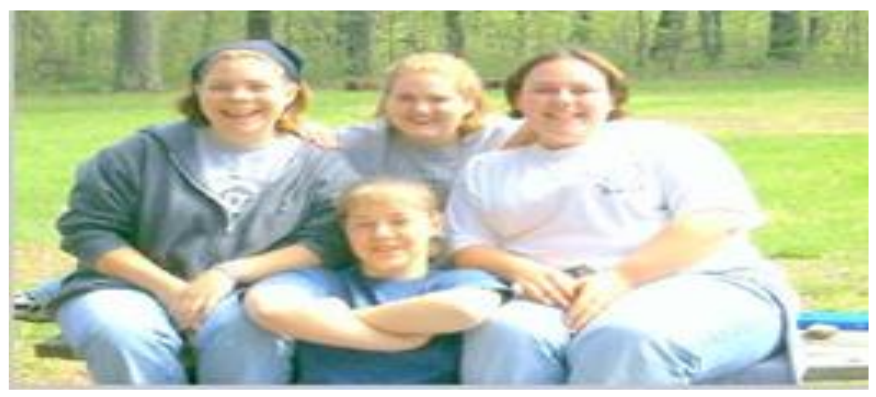

(b)

Fig. 8. Original images with complex background.

The results show that the developed algorithm is able to detect each of the faces despite the fact that they are of dissimilar frontal face sizes. Similarly, critical analysis of Fig. 9a and Fig. 9b show that the developed algorithm is capability of distinguishing faces from non-faces in an image with complex background. This capability also shows that occurrence of face(s) in a complex background has no negative impact on the detection accuracy of the developed face detection algorithm for this study.

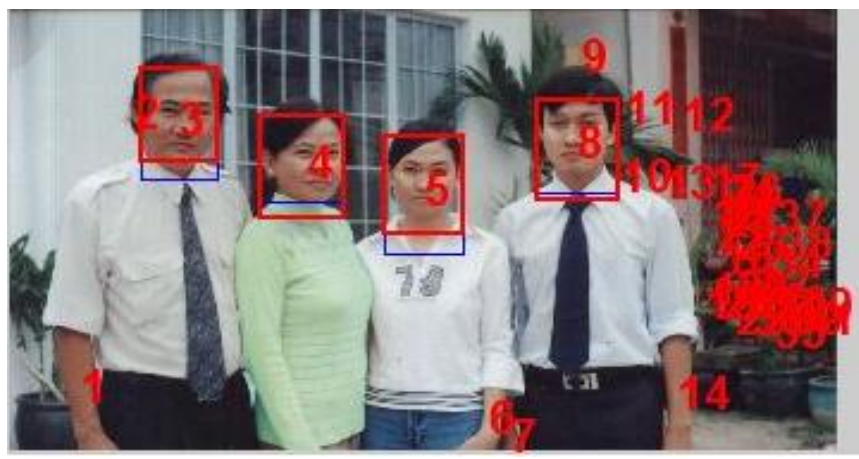

(a)

Fig. 9a. Face finding result for Fig. 8a.

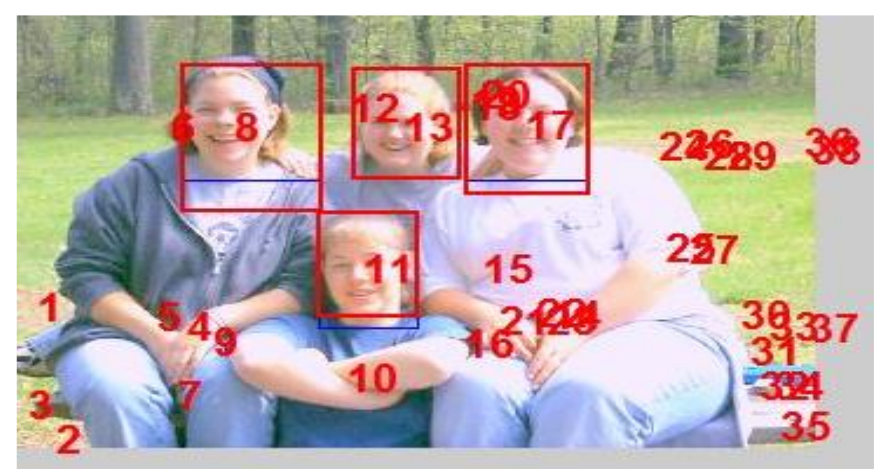

(b)

Fig. 9b. Face finding result from Fig. 8b.

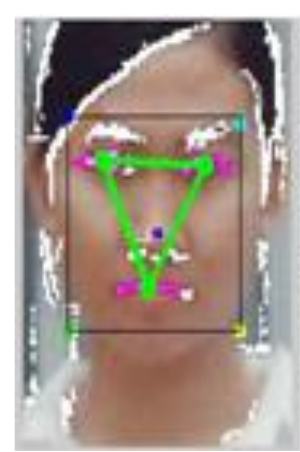

(c)

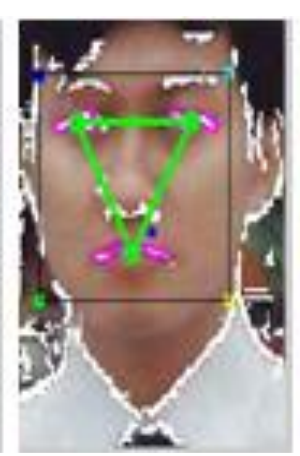

(c)

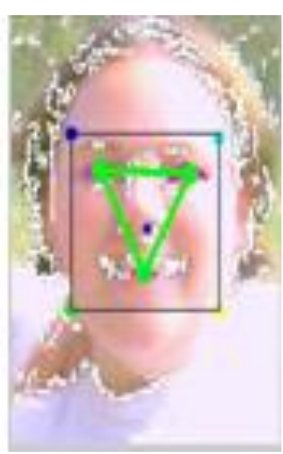

Fig. 9a and Fig. 9b. 

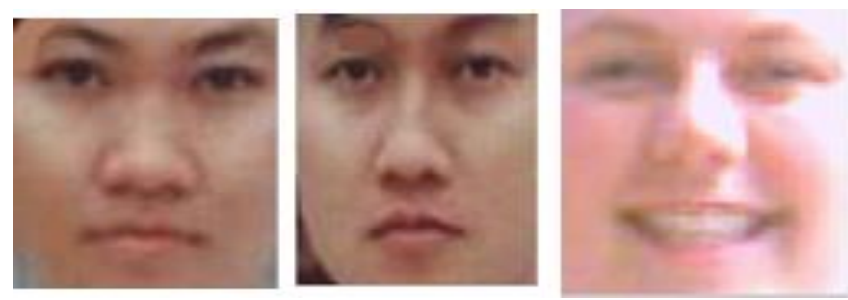

(d)

Fig. 9d. Some Detected faces Fig. 9c.

\subsection{Developed Algorithm Comparative Performance Evaluation}

In order to further evaluate the performance of the developed face detection algorithm, results obtained from it were compared with similar face detection algorithm developed using the same Bao database for testing. The direct comparison of the present work with other similar studies in the surveyed literature is presented in Table 2 . The choice of the reference studies, $[9,27,28]$ were characterized by using the same Baodatabase for the evaluation however, the reference studies employed different features and methods. The result of the comparative performance evaluation shows that only two out of the three studies used in the comparative performance evaluation outperform the present work while the present work outperforms the third reference study. Comparing the detection rate of the developed algorithm in this study with corresponding detection rates in the reference studies show that the algorithm developed in this study performs relatively well with similar studies in the surveyed literature

Furthermore, the performance of the developed algorithm for this study was further evaluated by comparing its computational time with other similar studies in literature. The results of the computational time taken for another three different algorithms on face detection in literature were compared with the computational time taken for the algorithm developed in this study. Although, the processors and the memories of the systems used in running those algorithms differ, the result of the computational time taken presented in Table 3 shows that the algorithm developed in this study has a lesser computational time taken of 2.46 seconds. The comparative computational time taken result shows that the algorithm developed for this study outperforms others despite the fact that it has the largest image size compared with reference studies in literature. This significant advantage is as a result of the fact that the algorithm for this study does not work as a classifier.

Table 2. Comparative detection result of the present work and other published studies

\begin{tabular}{|l|l|c|c|}
\hline Reference Author(s) & Feature and method employed & Testing Database & Detection rate (\%) \\
\hline Erdem et al (2011) [9] & $\begin{array}{l}\text { Haar features and skin colour base } \\
\text { classifier }\end{array}$ & Bao database & 88.48 \\
\hline See et al (2013) [27] & $\begin{array}{l}\text { Skin segmentation and edge } \\
\text { detection }\end{array}$ & Bao database & 84.70 \\
\hline $\begin{array}{l}\text { Khammari and } \\
\text { Chemesse (2013) [28] }\end{array}$ & $\begin{array}{l}\text { Neural Network and Gaussian } \\
\text { model }\end{array}$ & Bao database & 77.47 \\
\hline Developed Algorithm & $\begin{array}{l}\text { Skin segmentation and facial } \\
\text { feature extraction }\end{array}$ & 81.37 \\
\hline
\end{tabular}

Table 3. Comparative result of computational time of the present work and others

\begin{tabular}{|l|l|c|c|}
\hline Author(s) & Method Applied & $\begin{array}{l}\text { Image Size } \\
\text { Range }\end{array}$ & $\begin{array}{l}\text { Average } \\
\text { (second) }\end{array}$ \\
\hline Rowley et al (1999) [29] & $\begin{array}{l}\text { Neutral Network Based Face } \\
\text { Detection }\end{array}$ & 320 by 240 & 5.00 \\
\hline $\begin{array}{l}\text { Garcia and Delakis (2002) } \\
\text { [30] }\end{array}$ & Skin segmentation and edge detection & 352 by 288 & 4.00 \\
\hline Mohammed et al (2008) [31] & Neural Network and Gaussian model & $\begin{array}{l}63 \text { by } 180 \text { to } 200 \\
\text { by } 219\end{array}$ & 5.00 \\
\hline Developed Algorithm & $\begin{array}{l}\text { Skin segmentation and facial feature } \\
\text { extraction }\end{array}$ & $\begin{array}{c}189 \text { by } 219 \text { to } \\
320 \text { by } 408\end{array}$ & 2.00 \\
\hline
\end{tabular}

\section{Conclusion}

In this paper, we have successfully developed a face detection algorithm that utilizes skin segmentation and facial feature extraction method to decrease high FDR in face detection algorithms. Similarly, we also employed white balance correction to correct any change in image temperature at the point of acquiring image in order to improve the result of skin segmentation and the overall detection rate of the algorithm. In addition, the face detection algorithm for this study was developed without being trained as a classifier, which has considerably, reduces the computation complexity of the developed face detection algorithm in this study. Furthermore, the performance evaluation of the developed algorithm using Bao database, which is a standard face image detection database, shows 
that the developed algorithm performs satisfactorily well with relatively high CDR. Typical results of the performance evaluation of the developed algorithm shows that the developed algorithm performed satisfactorily well with $81.37 \%$ detection rate. Furthermore, the comparative performance evaluation test carried out on the developed algorithm shows that the developed algorithm performs favorably well with other algorithms in literature with better computational time.

\section{References}

[1] S. Zafeiriou, C. Zhang, and Z. Zhang, "A survey on face detection in the wild: past. present and future", Computer Vision and Image Understanding, vol. 138, pp. 1-24, 2015.

[2] J. Jin, B. Xu, X. Liu, Y. Wang, L. Cao, L. Han, B. Zhou, and M. Li, "A face detection and location method based on feature binding" Signal Processing: Image Communication, vol. 36, pp. 179-189, 2015.

[3] H. Pan, Y. Zhu, and L. Xia, "Efficient and accurate face detection using heterogeneous feature descriptors and feature selection", Computer Vision and Image Understanding, vol. 117, pp. 12-28, 2013.

[4] G. Onder, and A. Kayacik, "Multiview face detection using Gabor filters and support vector machine", Technical Report, IDE0852, Bachlor Thesis in Computer System Engineering, School of Science, Computer and Electrical Engineering, Halmsted University, Sweden, 2008. Online [Available]: https://www.divaportal.org/smash/get/diva2:239370/FULLTEXT01.pdf. Accessed on October 17, 2016.

[5] D. Ghimire, and J. Lee, "A robust face detection method based on skin color and edges", Journal of Information Process System, vol. 9, no. 1, pp. 141-156, 2013.

[6] C. Lin, and K-C. Fan, "Triangle-based approach to the detection of human face", Journal of Pattern Recognition, vol. 34, no. 6, pp. 1271-1284, 2001.

[7] M.R Mahmoodi,. and S.M. Sayedi, "A face detection based on kernel probability map", Computers and Electrical Engineering, vol. 46, pp. 205-216, 2015.

[8] Y. Ban, S-K. Kim, S. Kim, K-A. Toh, and S. Lee, "Face detection based on skin color likelihood", Pattern Recognition, vol. 47, pp. 1573-1585, 2014.

[9] C.E. Erdem, S. Ulukaya, A., Karaali, and A.T. Erdem, "Combining HAAR feature and skin colour based classifier for face detection", In Proceedings of IEEE International Conference on Acoustic Speech and Signal, Prague Congress Centre, Prague, Czech Republic, pp. 1497-1500, 22-27 May 2011.

[10] M-H. Yang, D.J. Kriegman, and N. Ahuja, "Detecting faces in lmages: A survey". IEEE Transactions On Pattern Analysis and Machine Intelligence, vol. 24, no. 1, pp. 34-58, 2002.

[11] S.K. Singh, D.S. Chauhan, M. Vasta, and R Singh., "A robust skin colour based face detection algorithm",
Tamkang Journal of Science and Engineering, vol. 6, no. 4, pp. 227-234, 2003.

[12] H-J. Lin, S-Y. Wang, S-H. Yen, and Y-T. Kao, "Face detection based on skin color segmentation and neural network", In proceedings of IEEE International Conference on Neural Networks and Brain, Beijing, China, pp. 1144-1149, 13-15 October 2005.

[13] Y. Wang, and B. Yuan, "A novel approach for human face detection from colour 1mages under complex background", Pattern Recognition, vol. 34, no. 10, pp. 1983-1992, 2001.

[14] M. Tayyab, and M.F. Zafar, "Face detection using 2D-discrete cosine transform and back propagation neural network", in Proceedings of IEEE Conference on Emerging Technologies, Islamabad, Pakistan, pp. 35-39, 19-20 October 2009.

[15] J. Ruan, and J. Yin, "Face detection based on facial features and linear support vector machines", In Proceedings of the International Conference on Communication Software and Networks, Chengdu, Sichuan, China, pp. 371-375, 27-28 Febuary 2009.

[16] X. Liu, G. Geng, and X. Wang, "Automatically face detection based on bp neural network and bayesian decision", in Proceedings of 6th IEEE International Conference on Natural Computation, Yantai, China, pp. 1590-1594, 10-12 August 2010.

[17] C. Aiping, P. Lian, T. Yaobin, and N. Ning, "Face detection technology based on skin color segmentation and template matching. IEEE 2nd International Workshop on Education Technology and Computer. Wuhan, China, pp. 708-711, 6-7 March 2010.

[18] Z. Li, L.Xue, and F. Tan, (2010). "Face detection in complex background based on skin color features and improved adaboost algorithms", IEEE International Conference on Progress in Informatics and Computing, Shanghai, China, pp. 723-727, 10-12 December 2010.

[19] E. Hjelmas, and B.K. Low, "Face detection: A survey", Computer Vision and Image Understanding, vol. 83, pp. 236-274, 2001.

[20] H. Zhu, S. Zhou, J. Wang, and Z. Yin, "An algorithm of pornographic 1mage detection", in Proceedings of the 4th IEEE International Conference on Image and Graphics. Chengdu, Sichuan, China, pp. 801-804. 22-24 August 2007.

[21] M.J. Taylor, and T. Morris, "Adaptive skin segmentation via feature-based face detection", in Proceedings of International Society for Optics and Photonics, (SPIE Photonics) Brussels, Belgium, 14-17 April 2014. Online [Available]: http://www.cs.man.ac.uk/ tmorris/pubs/AdaptSS_SPIE.p df. Accessed on 17 October 2016.

[22] R.C. Mat, S. Azmi, R. Daud, A.N. Zulkifli, and F.K. Ahmad, "Morpholocal operation on printed circuit board (PCB) reverse Engineering using MATLAB", in Proceedings of Knowledge Management International 
Conference and Exhibition, Legend Hotel Kuala Lumpur, Malaysia, pp. 529-533, 6-8 June 2006.

[23] D. Chudasama, T. Patel, and S. Joshi, "Image segmentation using morphological operations", International Journal of Computer Applications, vol. 117. no. 18, pp. 16-19, 2015.

[24] A. Khanparde, S. Reddy, and S. Ravipudi, "Face detection using color based segmentation and morphological processing - a case study", in Proceedings of the International Symposium on Computer Engineering and Technology, Mandi Gobindgarh, Punjab, India, pp. 147-151, 19-20 March 2010.

[25] C. Gürel, "Development of a face Recognition System", A Master of Science Thesis at the Atilim University, Ankara, Turkey, p. 81, 2011. Online [Available]: http://docplayer.net/2761592-Developmentof-a-face-recognition-system-a-thesis-submitted-to-thegraduate-school-of-natural-and-applied-sciences-atilimuniversity-cahit-gurel.html. Accessed on October 20, 2016.

[26] H. Rahman, nd J. Afrin, "Human face detection in colour 1mages with complex background using triangular approach", Global Journal of Computer Science and Technology Graphics and Vision, vol. 13, no. 4, pp. 45$50,2013$.

[27] Y.C. See, N.M. Noor, and A.C. Lai, "Hybrid face detection with skin segmentation and edge detection", in
Proceedings of 3rd IEEE International Conference on Signal and Image Processing Applications. Melaka, Malaysia, pp. 406-411, 8-10 October 2013.

[28] M. Khammari, and B. Chemesse, "Face detection in complex background of colour 1mages using mixture gaussian model and neural network", International Conference on System and Processing Information., Guelma, Algeria, 12 - 14 May 2013. Online [Available]: https://www.researchgate.net/profile/Chemesse_ennehar _Bencheriet/publication/257945701_Face_Detection_in Complex_Background_of Color_Images_Using_Mixtur e_Gaussian_Model and_Neural_Network/links/02e7e53 314aca3ee57000000.pdf. Accessed on October 17, 2016.

[29] H. Rowley, S. Baluja, and T. Kanade, "Neural network-based face detection", IEEE Transactions on Pattern Analysis and Machine intelligence, vol. 20, no. 1, pp. 23-38, 1998.

[30] C. Garcia, and M. Delakis, "A neural network architecture for fast and robust face detection", IEEE 16th International Conference on Pattern Recognition, Quebec City, Canada, vol. 2, pp. 44-47, 11-15 August 2002.

[31] A. Mohamed, Y.W. Weng, J. Jiang, and S. Ipson, (2008). Face Detection Based Neural Networks Using Robust Skin Segmentation. In Proceedings of the 5th IEEE International conference on Multi-Systems, Signals and Devices. Amman, Jordan, pp. 1-5, 20-22 July 2008. 